\title{
Leukemia and lymphoma: a cost of doing business for adaptive immunity
}

\author{
Mark S. Schlissel, ${ }^{1}$ Chris R. Kaffer, and John D. Curry \\ Division of Immunology, Department of Molecular and Cell Biology, University of California at Berkeley, \\ Berkeley, California 94720, USA
}

\begin{abstract}
According to the old adage, "you don't get something for nothing." This is certainly the case for the novel genetic mechanisms involved in adaptive immunity-V(D)J recombination, class-switch recombination (CSR), and somatic hypermutation (SHM). These mechanisms allow animals to respond with great specificity to a seemingly limitless array of rapidly evolving microbes and their products while investing a relatively small amount of genetic capacity. The cost, however, is genomic instability and its potential contribution to malignancy. A majority of B- and T-cell leukemias and lymphomas are associated with chromosomal abnormalities that bear the hallmarks of aberrant V(D)J or class-switch recombination. In addition, there are examples of B-cell lymphomas with point mutations in proto-oncogenes consistent with a role for SHM. Recent work has led to an increasingly detailed understanding of the biochemistry of V(D)J recombination, CSR, and SHM. Attention is now being focused on precisely how these reactions contribute to genomic instability and cancer. A report by Reddy et al. (2006) in this issue of Genes \& Development describes the use of a novel assay to measure the frequency of DNA transposition that occurs as a byproduct of V(D)J recombination. This and other approaches should allow for the identification of conditions that predispose people to leukemia and lymphoma and the genetic pathways that minimize this risk.
\end{abstract}

\section{Novel mechanisms regulating antigen receptor genes in lymphocytes}

Unlike any other genes in metazoans, immunoglobulin $(I g)$ and T-cell receptor (TCR) genes are encoded in segments that must be recombined during B- and T-cell development to generate complete genes (Jung and Alt 2004). In the murine Ig heavy-chain (HC) locus, for example, there are $\sim 110 \mathrm{~V}_{\mathrm{H}}$ gene segments, $13 \mathrm{D}_{\mathrm{H}}$ gene segments, and four $\mathrm{J}_{\mathrm{H}}$ gene segments dispersed over $\sim 2.5$ $\mathrm{Mb}$ of subtelomeric DNA on chromosome 12. Each re-

${ }^{1}$ Corresponding author.

E-MAIL mss@berkeley.edu; FAX (510) 642-6845.

Article is online at http://www.genesdev.org/cgi/doi/10.1101/gad.1446506. arranging gene segment is flanked by a recombination signal sequence (RSS) that serves as the recognition element for the V(D)J recombinase (Fig. 1). The RSS consists of a conserved heptamer that immediately abuts the gene segment, a spacer of either 12 or 23 nucleotides, and a conserved nonamer. Only gene segments flanked by RSSs with dissimilar spacer lengths can efficiently rearrange with one another. RAG1 and RAG2 are the essential, lymphoid-specific components of the $\mathrm{V}(\mathrm{D}) \mathrm{J}$ recombinase. They form a complex with one another that recognizes 12/23 RSS pairs and introduces double-strand DNA (dsDNA) breaks precisely at the heptamer-coding element borders (Gellert 2002). These broken DNA molecules are then joined to form coding and signal joints with the involvement of the nonhomologous end-joining (NHEJ) group of DNA break-repair proteins expressed in all cells (Mills et al. 2003). Pairing of the products of rearranged IgHC and light-chain $(L C)$ or TCR $\alpha$ - and $\beta$ - or $\gamma$ - and $\delta$-chain genes results in generation of the enormous repertoire of antigen recognition molecules that fuels adaptive immunity.

Two other novel genetic mechanisms further modify Ig genes during a B-cell immune response. An individual variable domain can be expressed as part of an IgHC along with a number of different constant domains. This is accomplished by a second, distinct DNA rearrangement reaction known as CSR (Maizels 2005). In CSR, recombination occurs between switch sequences that are found $5^{\prime}$ to each set of constant region exons distributed over $200 \mathrm{~Kb}$ of DNA on the centromeric side of the IgHC locus. Switch sequences consist of $2-10 \mathrm{~Kb}$ of poorly conserved repetitive G-rich DNA. As is the case with $\mathrm{V}(\mathrm{D}) \mathrm{J}$ recombination, intervening DNA sequences are excised from the chromosome during CSR in the form a circular DNA molecule, but the location of the recombination event is not precise. Finally, B cells responding to antigen activate somatic hypermutation, a process that results in a striking, targeted increase in mutation frequency within the $I g H C$ and $L C$ variable exons (Li et al. 2004). Both CSR and SHM depend on the activity of the activation-induced deaminase (AID) gene that encodes a cytidine deaminase. Expression of this protein is hypothesized to result in the generation of DNA nicks and breaks in switch sequences and in se- 
Schlissel et al.

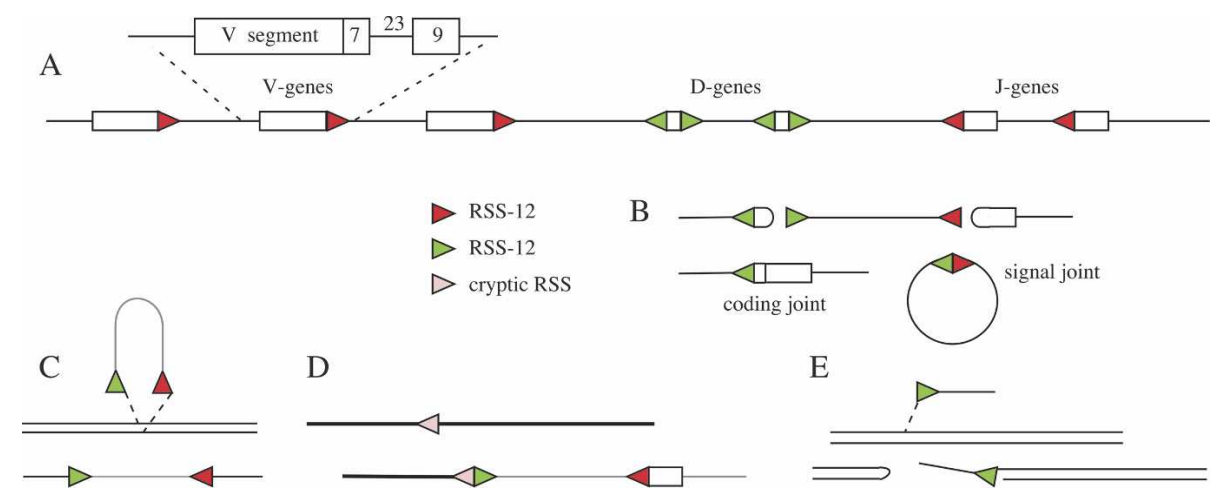

Figure 1. $\mathrm{V}(\mathrm{D}) \mathrm{J}$ recombination can result in DNA transposition and translocation. (A) Diagrammatic representation of the $I g H C$ locus showing a subset of $\mathrm{V}_{\mathrm{H}}, \mathrm{D}_{\mathrm{H}}$, and $\mathrm{J}_{\mathrm{H}}$ gene segments and their associated RSSs (triangles). The structure of a typical RSS is shown above. Intersegment distances are not to scale. $(B)$ Reaction intermediates (hairpin coding ends and blunt signal ends) and products (coding and signal joints). (C) Transposition of a RSS-ended recombination reaction intermediate. $(D)$ Rearrangement of a RSS-12 to a cryptic RSS resulting in a translocation. (E) Attack of a RSS on duplex DNA followed by transesterification.

quences subject to SHM, leading to recombination or mutation (Honjo et al. 2005).

\section{Chromosomal translocations and lymphoid malignancy}

Although leukemia, lymphoma, and myeloma are generally considered to be relatively rare diseases, taken together they constitute the fourth most common form of cancer. According to the National Cancer Institute, 4000 new cases of lymphocytic leukemia and 60,000 cases of lymphoma are diagnosed in the United States each year. To place this in context, a total of $\sim 1.3$ million cases of cancer were diagnosed in 2001, including $\sim 200,000$ each of lung, breast, and prostate cancers. Lymphoid malignancies are characterized by the frequent occurrence of chromosomal abnormalities, often translocations between proto-oncogenes and Ig or TCR loci (Table 1; Kuppers and Dalla-Favera 2001). Many of the most "famous" oncogenes, including $A b l, b c l-1$, and $b c l$ 2 , for example, were in fact discovered because they are located at translocation breakpoints in leukemias or lymphomas. The essential role of recombination in $\mathrm{Ig}$ and TCR gene expression led to the obvious hypothesis that some of these translocation events may actually represent the consequences of misregulated $\mathrm{V}(\mathrm{D}) \mathrm{J}$ recombination or CSR.

The first translocation to be associated with the V(D)J recombinase was $\mathrm{t}(14: 18)$, which joins a JH gene segment to the $B c 12$ proto-gene, giving rise to follicular lymphoma (and occasionally pre-B-cell leukemia) (Tsujimoto et al. 1985). Discovery of the genes involved in a large variety of other translocations involving antigen receptor gene segments followed thereafter. Not all $\mathrm{V}(\mathrm{D})$ J-like chromosome rearrangements involve antigenreceptor loci. The interstitial deletion $\mathrm{d}(1 \mathrm{p})$ that is associated with acute T-cell leukemia involves "cryptic" RSSs in two genes, TAL1 and SIL, which rearrange forming the SIL-TAL1 fusion product (Aplan et al. 1990). A $\mathrm{V}(\mathrm{D}) \mathrm{J}$ recombinase-mediated event deletes $\sim 90 \mathrm{~Kb}$ of DNA separating these two genes. It is thought that the fusion product gives the cells bearing it a growth advantage.

Leder and colleagues (Taub et al. 1982) identified the first chromosomal translocation likely to involve the CSR machinery. It consists of the c-Myc gene rearranged to an IgHC switch region and results in Burkitt's lymphoma. Other important examples of proto-oncogenes involved in CSR-mediated translocations include $b c l-3$, bcl-6, and IRF-4. The translocations most often result in overexpression of the proto-oncogene due to the influence of IgHC enhancer sequences. Because of the less well-defined nature of the switch sequence, it is not clear whether dsDNA breaks in translocation partner genes are caused by the CSR machinery or by other adventitious sources of DNA damage.

Recent studies from the Dalla-Favera laboratory (Pasqualucci et al. 2001) have revealed that SHM is not limited to $I g$ gene variable exons. These workers detected high frequencies of point mutations in normal mature B cells in the bcl-6 and CD95 genes and in several proto-oncogenes in various B-cell lymphomas. The nonrandom pattern of these mutations was consistent with the tendency of the SHM machinery to induce mutations in a gradient downstream of the gene's promoter. Interestingly, the regions of these genes that contain mutations are the ones targeted when the same gene is involved in a chromosomal translocation. It was suggested that DNA breaks induced during SHM in proto-oncogenes may serve to promote chromosomal translocations with $I g$ genes containing dsDNA breaks in switch sequences (Kuppers and Dalla-Favera 2001).

This hypothesis is consistent with a recent report that AID overexpression in cultured primary B cells results in the frequent translocation of $c$-myc to the $I g \mu$ switch region (Ramiro et al. 2006). Interestingly, these workers found that a null mutation in the $\mathrm{Ku} 80$ gene that greatly diminishes proper switch recombination had no effect on $A I D$-dependent $c-M y c-I g \mu$ translocation. Similarly, p53-null mice that show normal CSR and $A T M^{-1-}$ mice that show only minimally diminished CSR both dis- 
Table 1. Representative translocations associated with lymphoid malignancy

\begin{tabular}{lcll}
\hline Type & Translocation & Fusion genes & References \\
\hline TCR RSS to cryptic RSS & $\mathrm{t}(7 ; 9)$ & TCR $\beta /$ tal2 & Tycko et al. 1989 \\
IgH RSS to locus without RSS & $\mathrm{t}(14 ; 18)$ & IgH/bcl2 & Tsujimoto et al. 1985 \\
Coding joint & $\mathrm{t}(1 ; 19)$ & $E 2 A / p b x 1$ & Wiemels et al. 2002 \\
& $\mathrm{t}(9 ; 11)$ & AF-9/ALL-1 & Negrini et al. 1993 \\
Interstitial RSS & Exon $2 / 3$ deletions & Hprt & Finette et al. 2002 \\
Deletion & $\mathrm{d}(1)$ & sil/tal1 & Aplan et al. 1990 \\
\hline
\end{tabular}

played greatly enhanced translocation in this assay system. The ATM result is particularly interesting in light of the fact that ATM patients show unusually high levels of interlocus $\mathrm{V}(\mathrm{D}) \mathrm{J}$ recombination (Kirsch and Lista 1997). Taken together, these observations suggest that AID activity can lead to the generation of DNA breaks in both switch sequences and proto-oncogenes, resulting in translocation via repair events that rely on pathways in addition to classical NHEJ.

\section{The $\mathrm{V}(\mathrm{D}) \mathrm{J}$ recombinase and genomic instability}

The initial steps of $\mathrm{V}(\mathrm{D}) \mathrm{J}$ recombination involve RSS recognition by RAG-1 and RAG-2 followed by nicking of RSSs at the heptamer-coding element border, exposing a reactive 3' hydroxyl (Gellert 2002). A direct nucleophilic attack by the 3 ' hydroxyl on the phosphodiester bond on the antiparallel strand then results in the formation of a covalently sealed hairpin coding end and a blunt signal end (Fig. 1). This step requires synapsis between RSS-12 and RSS-23 complexes. The dsDNA breaks are stabilized by inclusion in a RAG-dependent post-cleavage synaptic complex, to which NHEJ proteins including DNA-PKcs, $\mathrm{Ku} 70, \mathrm{Ku} 80, \mathrm{XRCC} 4, \mathrm{DNA}$ ligase IV, Artemis, and Cernunnos are recruited. Interestingly, in wild-type mice, recombinase-mediated dsDNA breaks fail to activate p53 but are recognized by the machinery that localizes ATM and phosphorylated histone H2AX to dsDNA breaks (Mills et al. 2003). During the joining phase of recombination, hairpin opening is mediated by the endonucleolytic activity of Artemis. Finally, the coding ends of the V(D)J gene segments, which eventually form the functional antigen receptor gene, are joined and the blunt signal ends are ligated to form closed, usually episomal signal circles that are eventually lost from the cell.

The ability of an RSS to be acted upon by the recombinase is regulated by chromatin structure. Recombinase-accessible chromatin structure is associated with transcription, DNA demethylation, and histone modification (Sen and Oltz 2006). Cryptic RSSs (sequences with homology with Ig and TCR RSSs) can, however, cooperate with bona fide RSSs to activate $\mathrm{V}(\mathrm{D}) \mathrm{J}$ recombination on transfected reporter constructs (Raghavan et al. 2001). Thus, any such RSS in an accessible chromatin structure may be targeted by the recombinase, resulting in chromosomal deletions and translocations. In an elegant series of recent papers, Raghavan, Lieber, and colleagues (Raghavan et al. 2004) demonstrated that the V(D)J re- combinase can catalyze translocations to loci that lack apparent cryptic RSSs. They showed that the major breakage region (Mbr) where the majority of $\mathrm{IgHC}$-toBcl-2 transposition break points occur can function as an RSS to target V(D)J-like RAG-dependent rearrangement events. This property is associated with the ability of this sequence to form a non-B structure that the RAGs can bind and cleave. These results support the idea that in addition to recognizing a specific sequence, the RSS, the RAG complex can behave as a structure-specific nuclease with pathogenic potential.

RSS cleavage is thought to occur in a pairwise fashion. Under physiologic salt conditions in vitro, the RAGs will bind and nick individual RSS-12 or RSS-23 substrates, but will only go on to generate dsDNA breaks upon formation of a synaptic complex involving both types of RSSs (Gellert 2002). In addition, the recombinase displays a very strong bias for rearrangement in cis-between gene segments on the same chromosome (Kirsch and Lista 1997). It is interesting to note that in the cancer-prone disorder ataxia-telangectasia, this bias is reduced and that "trans" rearrangements between antigen receptor loci on different chromosomes correlates with the existence of oncogenic translocations in patients. Recent work has revealed that rearranging loci undergo developmentally regulated chromosome condensation, juxtaposing distal V-region gene segments with the D-J region of the $\mathrm{IgHC}$ locus in cells undergoing V-to-DJ rearrangement (Sen and Oltz 2006). This "looping" was shown to be dependent on the transcription factor Pax5, but independent of RAG expression. Thus, the requirement for RSS synapsis prior to cleavage and the bias in favor of rearrangement in cis may serve to limit the likelihood that RAGs will introduce dsDNA breaks into individual cryptic RSSs in nonantigen receptor loci.

Two aspects of recombinase biochemistry heighten the risk of genomic instability, however. First, Roth and colleagues (Neiditch et al. 2002) showed that RSSs within signal joints can be cleaved by the recombinase, regenerating open, recombinogenic RSS ends so long as RAGs continue to be expressed. This observation provides an explanation for why free signal ends appear to persist in cells longer than coding ends (Gellert 2002). Second, our group recently found that RAGs will bind and nick multiple RSS-12 sequences in pre-B cells independent of synapsis and RSS cleavage (Curry et al. 2005). In light of the observation that $\mathrm{V}(\mathrm{D}) \mathrm{J}$ recombinase-mediated RSS nicking can activate homologous recombina- 
tion in vivo (Lee et al. 2004), these nicked intermediates might participate in the potentially mutagenic transfer of information in cells undergoing $\mathrm{V}(\mathrm{D}) \mathrm{J}$ recombination.

Null mutation of genes encoding NHEJ proteins causes a near-complete block in $\mathrm{V}(\mathrm{D}) \mathrm{J}$ recombination but does not result in frequent leukemia or lymphoma (Mills et al. 2003; Jung and Alt 2004). Some of these mutations are associated with embryonic lethality (Ligase IV and $X R C C 4)$, others cause runting and various organ abnormalities (Ku70), while DNA-PK and Artemis mutations have near-normal nonlymphoid phenotypes. Breeding these mutations onto a p53-null background, however, results in high frequencies of malignancy, most often pro-B-cell leukemia (Zhu et al. 2002). Almost all of these leukemic pro-B cells contain RAG-dependent IgHC-myc translocations and more complex chromosomal abnormalities termed complicons. While the $p 53$ mutation on an otherwise wild-type background predisposes to thymic lymphoma, these tumors are not RAG dependent and thus must involve other mechanisms. Similarly, although ATM-deficient mice and humans with ataxiatelangectasia are prone to lymphoid malignancy, the RAGs do not play an obligatory role. Thus, it is somewhat surprising that $H 2 A X$-null mice show a modest CSR defect but when combined with p53 deficiency develop leukemias and lymphomas that appear to involve V(D)J recombination or CSR (Bassing et al. 2003). In sum, these data suggest that genes involved in dsDNA break sensing or repair are required to protect the genome from the consequences of RAG- or CSR-induced DNA breaks. Given that the rate of production of lymphoid cells in humans is between $10^{7}$ and $10^{8}$ cells per day and that each cell must undergo multiple $\mathrm{V}(\mathrm{D}) \mathrm{J}$ recombination events to assemble its antigen receptor, it is remarkable how effective this machinery is at avoiding biologically significant mistakes.

It is interesting to consider why RAG-dependent translocations seem to target particular chromosomal sites. One possibility is that Ig and TCR locus translocations are quite frequent, but are only discovered when the partner locus can affect either proliferation or survival of lymphoid cells (i.e., these cells have a selective advantage). Another possibility is that certain translocation events are favored because of intranuclear juxtaposition of chromosomal domains. The recent discovery by Flavell and co-workers (Spilianakis et al. 2005) of developmentally regulated physical contact between the TH2 cytokine cluster on chromosome 11 and the Ifn- $\gamma$ locus on chromosome 10 provides a clear precedent. This hypothesis can be tested using either fluorescent in situ hybridization or biochemical approaches to assay for colocalization of known translocation partners with Ig or TCR loci in normal, developing lymphocytes.

\section{The $\mathrm{V}(\mathrm{D}) \mathrm{J}$ recombinase is a transposase}

Several lines of evidence suggest that RAG-1 and RAG-2 are derived from an ancient transposable element (Lewis and $\mathrm{Wu}$ 1997). The two genes are linked and located within $8 \mathrm{~Kb}$ of one another, they are convergently tran- scribed, and their coding regions lack introns. In addition, many biochemical studies of the V(D)J recombinase reaction have demonstrated similarities with transposases and transposition reactions (Jones and Gellert 2004). Like bacteriophage Mu and HIV integrase, hairpin formation during recombination occurs through a direct transesterification without covalent DNA intermediates. Similar to V(D)J recombination, Tn10 excision occurs through a covalently sealed hairpin intermediate. The active site of RAG- 1 contains a DDE acidic metal chelating triad, a motif required for activity of bacteriophage MuA, Tn7, and Tn10 transposase as well as the HIV-1 integrase.

Initial evidence that the $\mathrm{V}(\mathrm{D}) \mathrm{J}$ recombinase could, in fact, function as a transposase in vitro was provided by seminal studies from the Schatz and Gellert laboratories (Agrawal et al. 1998; Hiom et al. 1998). These workers found that in the presence of RAG proteins, RSS-12 and RSS-23 DNA ends undergo transposition reactions in which the $3^{\prime} \mathrm{OH}$ of signal ends attack either the same strand, resulting in deletion circles, or the antiparallel strand to form closed circles. Furthermore, intermolecular transposition events were observed in which DNA substrates flanked by RSSs integrated into a circular target plasmid. These insertions contained target site duplications, a hallmark of DNA transposition. Because transposition of exogenous DNA into regulatory or protein coding regions could be deleterious to cell survival, the suppression of transposition must be a highly stringent process. Studies from the Schatz laboratory (Tsai and Schatz 2003) have suggested that suppression of signal end transposition may be a property of the C-terminal domain of RAG2, which is not essential for V(D)J recombination.

The RAG complex possesses a number of other related biochemical activities that may contribute to genomic instability. Like transposition, these activities have to do with the ability of the RAGs to activate nucleophilic attack on duplex DNA by the 3' OH group on an RSS end. As pointed out previously, such strand-transfer reactions can be followed by so-called a transesterification reaction generating the equivalent of a hairpin coding end in a target locus (Fig. 1; Melek and Gellert 2000). This coding end could then be repaired by joining to a coding end within an antigen receptor locus. Such a mechanism could explain how the RAGs might be involved in translocations to target loci that lack cryptic RSSs.

Despite strong data supporting the ability of the RAGs to catalyze transposition in vitro, transposition of RSSended DNA fragments has been difficult to detect in vivo. The hprt locus is widely used in assays to detect and analyze mutation in cultured cells since it is located on the X-chromosome and its product can be selected against by culturing cells with 6-thioguanine (6-TG). Of the many hundreds of 6-TG resistant human peripheral $\mathrm{T}$-cell clones studied by numerous laboratories, two were found to be due to insertions of RSS-ended fragments from the TCR $\alpha$ locus (Messier et al. 2003). These insertions contained the target-site duplications characteristic of transposition. Interestingly, both occurred 
near previously identified cryptic RSSs in the first intron of hprt. Far more frequent mutations detected in hprt consist of deletions of DNA between cryptic RSSs in introns 1 and 3 (Finette et al. 1996).

\section{Detection and quantification of RSS fragment transposition}

It has proven challenging to set up an experimental system with which to analyze RAG-mediated genomic instability in vivo. One group established a V(D)J recombination system in yeast by introducing regulated RAG1 and RAG2 expression vectors and an episomal target containing a pair of RSSs (Clatworthy et al. 2003). While recombination itself was detectable but inefficient in this system, RSS fragment transposition was too rare to be analyzed in any meaningful detail (about one event per $10^{8}$ yeast cells).

A recent study from the Schatz laboratory (Chatterii et al. 2006) utilized an episomal reporter construct assay to detect RSS-fragment transposition in transiently transfected $293 \mathrm{~T}$ cells (a human embryonic kidney cell line). Their strategy relied on a transposition donor plasmid containing a tetracycline resistance gene flanked by RSS12 and RSS-23 sequences, flanked by two negative selection markers. A target plasmid lacking tetracycline resistance but containing a kanamycin resistance gene was cotransfected with the donor plasmid along with various RAG1 and RAG2 expression vectors. Two days after transfection, the episomes were isolated and transformed into bacteria, selecting for transfer of tetracycline resistance to the target plasmid. Very few of the recovered plasmids displayed the hallmarks of insertional transposition. Chatterji et al. (2006) estimated the frequency of transposition between these two episomes to be $\sim 1.0 \times 10^{-7}$. They went on to assay for transposition of RSS-ended fragments from a donor plasmid into chromososomal DNA in this system. Only two such events were recovered, and neither showed clear evidence of RAG-mediated transposition.

The paper in this issue of Genes \& Development from Reddy et al. (2006) represents a major step forward in this regard. These authors used retroviral transduction to integrate a single-copy reporter construct into the genome of a transformed pro-B-cell line that was inducible for high-frequency $\mathrm{V}(\mathrm{D}) \mathrm{J}$ recombination. The cleverly designed reporter allowed for the selection of cells that had undergone rearrangement, but had retained the excised RSS-flanked recombination intermediate. Of 21 recovered subclones, seven displayed clear evidence of insertional transposition, including target site duplication. They estimated the frequency of this event as 1 per 44,000 reporter construct rearrangements. Remarkably, two of these transpositions targeted switch sequences in the IgHC locus normally involved in CSR. While the authors suggested this might be due to secondary structure formation in these repetitive GC-rich sequences, it is also be possible that the cell line used in these studies expressed low levels of AID. If this were the case, AID might generate DNA damage in these switch sequences, contributing to their capture of RSS-ended recombination intermediates. Many of the 14 other characterized transpositions occurred into Ig HC or LC loci and involved DNA breaks in these targets introduced by $\mathrm{V}(\mathrm{D}) \mathrm{J}$ recombination.

It is possible that this experimental system overestimates the frequency of transposition events due to the absence of important, cis-acting sequences. For example, promoters upstream and enhancers downstream of rearranging antigen receptor gene segments may play a role in appropriate RSS synapsis. Chromosomal structure might also serve to limit transposition if rearranging loci occupy defined domains within the nucleus. In addition, the size of the excised, RSS-ended fragment (difficult to determine from the paper by Reddy et al. [2006]) may play a role in its fate. The distances between naturally rearranging pairs of RSSs are either quite short $(<1-3 \mathrm{~Kb})$ or extremely long $(>20 \mathrm{~Kb})$. It is possible that intermediate lengths are more compatible with transposition and that inter-RSS distances have undergone evolutionary selection on this basis. If the estimated frequency of one in 44,000 recombination events is correct, however, the genomes of our lymphoid compartment must be littered with the detritus of $\mathrm{V}(\mathrm{D}) \mathrm{J}$ recombination. One can imagine experimental approaches to search for events involving native RSS-ended transposons in normal cells.

These workers are now in an excellent position to identify cellular components that serve to suppress RSS fragment transposition by using their reporter system in transformed pro-B-cell lines generated from NHEJ and other DNA repair and checkpoint-deficient mutant mice. Insights from such studies might lead to a search for genetic polymorphisms or environmental exposures that heighten the risk of recombinase-mediated genomic instability in humans.

\section{Acknowledgments}

We acknowledge useful discussions with Dr. Jef Boeke (Johns Hopkins Medical School) and Fred Alt (Harvard Medical School), and generous research support from the NIH.

\section{References}

Agrawal, A., Eastman, Q.M., and Schatz, D.G. 1998. Transposition mediated by RAG1 and RAG2 and its implications for the evolution of the immune system. Nature 394: 744-751.

Aplan, P.D., Lombardi, D.P., Ginsberg, A.M., Cossman, J., Bertness, V.L., and Kirsch, I.R. 1990. Disruption of the human SCL locus by 'illegitimate' V-(D)-J recombinase activity. Science 250: 1426-1429.

Bassing, C.H., Suh, H., Ferguson, D.O., Chua, K.F., Manis, J., Eckersdorff, M., Gleason, M., Bronson, R., Lee, C., and Alt, F.W. 2003. Histone H2AX: A dosage-dependent suppressor of oncogenic translocations and tumors. Cell 114: 359-370.

Chatterii, M., Tsai, C.L., and Schatz, D.G. 2006. Mobilization of RAG-generated signal ends by transposition and insertion in vivo. Mol. Cell. Biol. 26: 1558-1568.

Clatworthy, A.E., Valencia, M.A., Haber, J.E., and Oettinger, M.A. 2003. V(D)J recombination and RAG-mediated transposition in yeast. Mol. Cell 12: 489-499. 
Curry, J.D., Geier, J.K., and Schlissel, M.S. 2005. Single-strand recombination signal sequence nicks in vivo: Evidence for a capture model of synapsis. Nat. Immunol. 6: 1272-1279.

Finette, B.A., Poseno, T., and Albertini, R.J. 1996. V(D)J recombinase-mediated HPRT mutations in peripheral blood lymphocytes of normal children. Cancer Res. 56: 1405-1412.

Finette, B.A., Kendall, H., and Vacek, P.M. 2002. Mutational spectral analysis at the HPRT locus in healthy children. Mutat. Res. 505: 27-41.

Gellert, M. 2002. V(D)J recombination: RAG proteins, repair factors, and regulation. Annu. Rev. Biochem. 71: 101-132.

Hiom, K., Melek, M., and Gellert, M. 1998. DNA transposition by the RAG1 and RAG2 proteins: A possible source of oncogenic translocations. Cell 94: 463-470.

Honjo, T., Nagaoka, H., Shinkura, R., and Muramatsu, M. 2005. AID to overcome the limitations of genomic information. Nat. Immunol. 6: 655-661.

Jones, J.M. and Gellert, M. 2004. The taming of a transposon: $\mathrm{V}(\mathrm{D}) \mathrm{J}$ recombination and the immune system. Immunol. Rev. 200: 233-248.

Jung, D. and Alt, F.W. 2004. Unraveling V(D)|J recombination; insights into gene regulation. Cell 116: 299-311.

Kirsch, I.R. and Lista, F. 1997. Lymphocyte-specific genomic instability and risk of lymphoid malignancy. Semin. Immunol. 9: 207-215

Kuppers, R. and Dalla-Favera, R. 2001. Mechanisms of chromosomal translocations in B cell lymphomas. Oncogene 20: $5580-5594$.

Lee, G.S., Neiditch, M.B., Salus, S.S., and Roth, D.B. 2004. RAG proteins shepherd double-strand breaks to a specific pathway, suppressing error-prone repair, but RAG nicking initiates homologous recombination. Cell 117: 171-184.

Lewis, S.M. and Wu, G.E. 1997. The origins of V(D)J recombination. Cell 88: 159-162.

Li, Z., Woo, C.J., Iglesias-Ussel, M.D., Ronai, D., and Scharff, M.D. 2004. The generation of antibody diversity through somatic hypermutation and class switch recombination. Genes \& Dev. 18: 1-11.

Maizels, N. 2005. Immunoglobulin gene diversification. Annu. Rev. Genet. 39: 23-46.

Melek, M. and Gellert, M. 2000. RAG1/2-mediated resolution of transposition intermediates: Two pathways and possible consequences. Cell 101: 625-633.

Messier, T.L., O'Neill, J.P., Hou, S.M., Nicklas, J.A., and Finette, B.A. 2003. In vivo transposition mediated by $\mathrm{V}(\mathrm{D}) \mathrm{J}$ recombinase in human T lymphocytes. EMBO J. 22: 13811388.

Mills, K.D., Ferguson, D.O., and Alt, F.W. 2003. The role of DNA breaks in genomic instability and tumorigenesis. Immunol. Rev. 194: 77-95.

Negrini, M., Felix, C.A., Martin, C., Lange, B.J., Nakamura, T., Canaani, E., and Croce, C.M. 1993. Potential topoisomerase II DNA-binding sites at the breakpoints of a $t(9 ; 11)$ chromosome translocation in acute myeloid leukemia. Cancer Res. 53: 4489-4492.

Neiditch, M.B., Lee, G.S., Huye, L.E., Brandt, V.L., and Roth, D.B. 2002. The V(D)J recombinase efficiently cleaves and transposes signal joints. Mol. Cell 9: 871-878.

Pasqualucci, L., Neumeister, P., Goossens, T., Nanjangud, G., Chaganti, R.S., Kuppers, R., and Dalla-Favera, R. 2001. Hypermutation of multiple proto-oncogenes in B-cell diffuse large-cell lymphomas. Nature 412: 341-346.

Raghavan, S.C., Kirsch, I.R., and Lieber, M.R. 2001. Analysis of the $\mathrm{V}(\mathrm{D}) \mathrm{J}$ recombination efficiency at lymphoid chromosomal translocation breakpoints. J. Biol. Chem. 276: 2912629133.
Raghavan, S.C., Swanson, P.C., Wu, X., Hsieh, C.L., and Lieber, M.R. 2004. A non-B-DNA structure at the $B c l-2$ major breakpoint region is cleaved by the RAG complex. Nature 428: 88-93.

Ramiro, A.R., Jankovic, M., Callen, E., Difilippantonio, S., Chen, H.T., McBride, K.M., Eisenreich, T.R., Chen, J., Dickins, R.A., Lowe, S.W., et al. 2006. Role of genomic instability and p53 in AID-induced $c$-myc-Igh translocations. Nature 440: 105-109.

Reddy, Y.V.R., Perkins, E.J., and Ramsden, D.A. 2006. Genomic instability due to $\mathrm{V}(\mathrm{D}) \mathrm{J}$ recombination-associated transposition. Genes \& Dev. (this issue).

Sen, R. and Oltz, E. 2006. Genetic and epigenetic regulation of IgH gene assembly. Curr. Opin. Immunol. 18: 237-242.

Spilianakis, C.G., Lalioti, M.D., Town, T., Lee, G.R., and Flavell, R.A. 2005. Interchromosomal associations between alternatively expressed loci. Nature 435: 637-645.

Taub, R., Kirsch, I., Morton, C., Lenoir, G., Swan, D., Tronick, S., Aaronson, S., and Leder, P. 1982. Translocation of the c-myc gene into the immunoglobulin heavy chain locus in human Burkitt lymphoma and murine plasmacytoma cells. Proc. Natl. Acad. Sci. 79: 7837-7841.

Tsai, C.L. and Schatz, D.G. 2003. Regulation of RAG1/RAG2mediated transposition by GTP and the C-terminal region of RAG2. EMBO I. 22: 1922-1930.

Tsujimoto, Y., Gorham, J., Cossman, J., Jaffe, E., and Croce, C.M. 1985. The $t(14 ; 18)$ chromosome translocations involved in B-cell neoplasms result from mistakes in VDJ joining. Science 229: 1390-1393.

Tycko, B., Reynolds, T.C., Smith, S.D., and Sklar, J. 1989. Consistent breakage between consensus recombinase heptamers of chromosome 9 DNA in a recurrent chromosomal translocation of human T cell leukemia. J. Exp. Med. 169: 369377.

Wiemels, J.L., Leonard, B.C., Wang, Y., Segal, M.R., Hunger, S.P., Smith, M.T., Crouse, V., Ma, X., Buffler, P.A., and Pine, S.R. 2002. Site-specific translocation and evidence of postnatal origin of the $t(1 ; 19) E 2 A-P B X 1$ fusion in childhood acute lymphoblastic leukemia. Proc. Natl. Acad. Sci. 99: 15101-15106.

Zhu, C., Mills, K.D., Ferguson, D.O., Lee, C., Manis, J., Fleming, J., Gao, Y., Morton, C.C., and Alt, F.W. 2002. Unrepaired DNA breaks in p53-deficient cells lead to oncogenic gene amplification subsequent to translocations. Cell 109: 811821. 


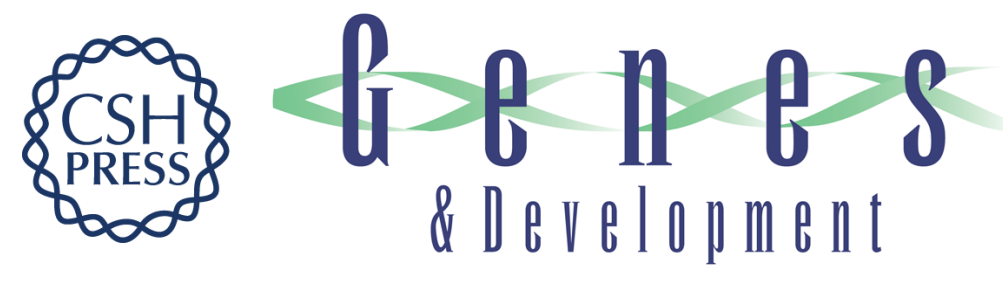

\section{Leukemia and lymphoma: a cost of doing business for adaptive immunity}

Mark S. Schlissel, Chris R. Kaffer and John D. Curry

Genes Dev. 2006, 20:

Access the most recent version at doi:10.1101/gad.1446506
Related Content Genomic instability due to $V(D) J$ recombination-associated transposition
Yeturu V.R. Reddy, Eric J. Perkins and Ramsden
Genes Dev. UNKNOWN , 2006 20: 1575-1582
References This article cites 36 articles, 12 of which can be accessed free at:
http://genesdev.cshlp.org/content/20/12/1539.full.html\#ref-list-1
Articles cited in:
http://genesdev.cshlp.org/content/20/12/1539.full.html\#related-urls

\section{License}
Email Alerting Receive free email alerts when new articles cite this article - sign up in the box at the top Service right corner of the article or click here.

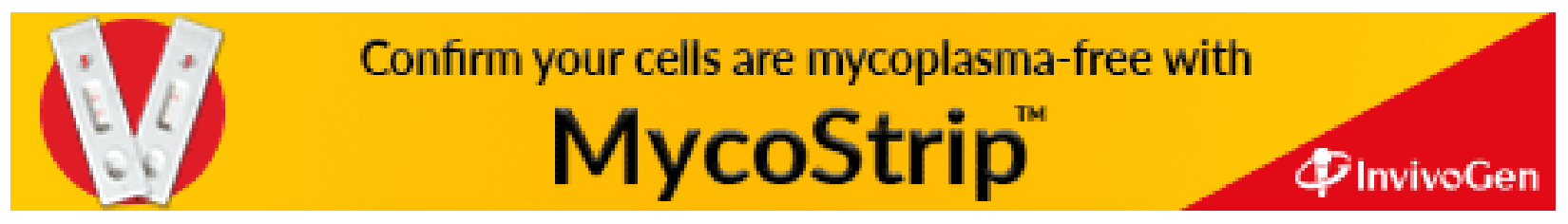

